\title{
Optical Tests on a Curve Fresnel Lens as Secondary Optics for Solar Troughs
}

\author{
D. Fontani, ${ }^{1}$ P. Sansoni, ${ }^{1}$ F. Francini, ${ }^{1}$ M. DeLucia, ${ }^{2}$ G. Pierucci, ${ }^{2}$ and D. Jafrancesco ${ }^{1}$ \\ ${ }^{1}$ CNR-National Institute of Optics (INO), Largo E. Fermi, 6, 50125 Firenze, Italy \\ ${ }^{2}$ Department DIEF, University of Florence, Via Santa Marta, 3, 50139 Firenze, Italy \\ Correspondence should be addressed to P. Sansoni; paola.sansoni@ino.it
}

Received 30 May 2017; Accepted 9 July 2017; Published 12 September 2017

Academic Editor: Prakash Basnyat

Copyright (c) 2017 D. Fontani et al. This is an open access article distributed under the Creative Commons Attribution License, which permits unrestricted use, distribution, and reproduction in any medium, provided the original work is properly cited.

\begin{abstract}
A curve Fresnel lens is developed as secondary concentrator for solar parabolic troughs to reduce the number of photovoltaic cells. Specific measurements and optical tests are used to evaluate the optical features of manufactured samples. The cylindrical Fresnel lens transforms the focal line, produced by the primary mirror, into a series of focal points. The execution of special laboratory tests on some secondary concentrator samples is discussed in detail, illustrating the methodologies tailored to the specific case. Focusing tests are performed, illuminating different areas of the lens with solar divergence light and acquiring images on the plane of the photocell using a CMOS camera. Concentration measurements are carried out to select the best performing samples of curve Fresnel lens. The insertion of the secondary optics within the concentrating photovoltaic (CPV) trough doubles the solar concentration of the system. The mean concentration ratio is $1.73,2.13$, and 2.09 for the three tested lenses. The concentration ratio of the solar trough is 140 and approaches 300 after the introduction of the secondary lens.
\end{abstract}

\section{Introduction}

Optical characterization and practical experimentation on manufactured components are key elements to address and ameliorate the production process and to select the best performing samples. When the optical component presents a particular geometry of radiation collection, the tests need to be tailored to examine it. Furthermore, specific measurement procedures and customized set-ups can study peculiarities or interesting aspects of the examined samples. Optical tests are frequently used to identify defective portions of the realized samples to correct manufacture.

The tested component is a novel secondary optics especially designed and implemented for an existing concentrating photovoltaic (CPV) trough. It is a cylindrical prismatic lens of Fresnel type [1]. This secondary concentrator reduces the number of photovoltaic (PV) cells in the solar trough [2-6], which is used for the combined production of heat and electricity [7, 8]. The examined parabolic trough combines photovoltaic systems and thermal devices: PV cells directly exploit the sunlight concentrated on them, and their cooling system supplies thermal energy.

The linear parabolic reflector (primary mirror) acts only in the direction transversal to the solar trough, so the entire focal line must be covered by a cell row [2]. The proposed secondary optics is interposed between the linear parabolic mirror and the linear row of PV cells $[9,10]$. The sunlight collected by the parabolic mirror is intercepted by the secondary optics and concentrated, in portions, along the longitudinal axis of the solar trough.

The working principle of the cylindrical Fresnel lens (CFL) is that it transforms the focal line, concentrated by the linear parabolic mirror, into a series of focal points (Figure 1). Hence, the proposed solution allows to reduce the number of PV cells because it increases the sunlight concentration. In practice, the production of electricity is not affected by the reduction of cell number, because it is balanced by an enhancement of solar concentration.

The research started with the optical design of the secondary component [1], selecting a configuration that optimizes collection efficiency of the whole system and 


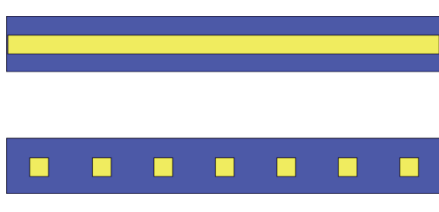

Figure 1: The cylindrical Fresnel lens (CFL) reconcentrates the radiation on a square photocell.

uniformity of the PV cell illumination. The optical design [11] of this secondary concentrator must take into account the characteristics of the existing trough and of the photovoltaic cells $[9,10]$. The optical parameters of the secondary optics are principally determined by examining the irradiance distribution on the receiver $[9,10]$. Analyses and simulations of the effects due to collector deformation, axial defocusing, tracking errors, and receiver alignment errors completed the study [1]. Some auxiliary analyses optimized operative conditions and collection efficiency of the whole system, in order to maintain elevated performance of the solar trough in real working conditions.

The solar installation is not a classical linear trough but is an innovative device provided with a two-axis tracking system that permits to follow the sun and to keep the receiver always aligned. Therefore, the insertion of a secondary element does not require to modify the original tracker.

In practice, the curve Fresnel lens was specifically designed for a trough, whose parabolic mirror focuses the light over an articulated absorber. The focal image is a rectangle, and it is concentrated on a row of squared photovoltaic cells $[9,10]$. The cells are placed on a metallic tube of rectangular section, which acts as cooling system by means of a liquid flowing inside. An external protection tube in glass, enclosing cells and cooler, completes the absorber (Figure 2). The photocells directly exploit the concentrated sunlight providing electricity, while the cooling system of the cells furnishes heat. In this concentration geometry, the curve Fresnel lens must be located inside the parabolic mirror around and near the absorber, constituted by a glass tube enclosing a row of photovoltaic cells placed over a rectangular tube. The receiver photo in Figure 2 shows the cell row before the insertion of the cylindrical Fresnel lens that will reduce the number of photocells. The system concentration ratio before the introduction of the secondary optics was 140. The improvement of concentration ratio is not so great to require a change in cell type. The concentration is double, so it can be managed by the original cells.

The next phase was the practical implementation of the cylindrical Fresnel lens. Successive series of samples were manufactured, improving the quality of the production by adjusting the optical realization parameters. To check the optical quality of the realized samples, they were optically characterized in laboratory. The test procedures were especially tailored taking into account collection geometry and particular shape of the curve Fresnel lens. Some of these tests and measurements are illustrated in the next sections, presenting as exemplificative results the data measured on three lenses of the last production of CFLs.

\section{Collection Geometry}

Based on the optical design of the cylindrical Fresnel lens (CFL), developed as secondary optics for parabolic troughs, some samples were implemented by molding production. The research proceeded with the optical characterization of the samples by means of tests to evaluate the accuracy of the realized shape of the CFLs. These optical tests are useful both to control the progresses achieved by the successive series of mass production and to select the best performing samples among the realized optical components.

The examined solar trough uses a parabolic trough concentrator (PTC) and a series of cylindrical Fresnel lenses that concentrate solar radiation in two orthogonal directions. Figure 1 illustrates the action of a row of CFLs. The PTC concentrates the sunlight (in transversal direction) on the receiver tube; then the CFLs are interposed between the tube and the PTC and reconcentrate the light. The coupled concentrators (PTC and CFL) are optically designed to illuminate a PV cell of $10 \times 10 \mathrm{~mm}$ size. In practice, the CFLs are used in the same number as the PV cells mounted on the tube and the action of the lens row is to transform the focal line into a row of focal points.

As can be seen from Figure 3, the CFL has cylindrical symmetry. Concentration takes place in both directions: Figure 3 shows the directions of light concentration. The lens is completely illuminated in the longitudinal direction (red arrow); while in the transversal axis (blue arrow), where the PTC concentrates, the rays arrive on the cell within an aperture angle of about $\pm 50^{\circ}$ (Figure 4(a)). The red arrow direction coincides with the axis of the cylinder of the curve Fresnel lens. The pictures in Figure 4 show top and lateral views of the CFL inserted into the trough. In Figure 4(b), the green rays are those reflected by the parabolic mirror, while the blue ones are the rays arriving from the sun; for clarity, the tube has not been drawn.

The next sections present tailored methodologies and results of laboratory tests on prototypes of these cylindrical Fresnel lenses. The purpose of the optical tests is to evaluate the quality of the production process by testing the optical behavior of the samples in terms of concentration factor and image quality. To perform these tests, a set-up has been developed to separately illuminate different lens sectors in order to analyze their optical features.

\section{Tailored Optical Tests: Image Analysis}

The test [12] performed on the CFL samples used a solar divergence collimator and a CMOS camera.

A solar divergence collimator [13] produces a beam with solar divergence of about $250 \mathrm{~mm}$ in diameter, with a high uniformity. Choosing appropriate components and set-up, the value of solar divergence results 0.48 degrees (total angular aperture, $2 \theta$ ).

The beam was used directly to illuminate the CFL sample. A vertical diaphragm (Figure 5) was placed between the mirror of the solar divergence collimator and the CFL; in this way, the lens is fully illuminated in longitudinal direction, while in the transversal direction, the illumination is limited 


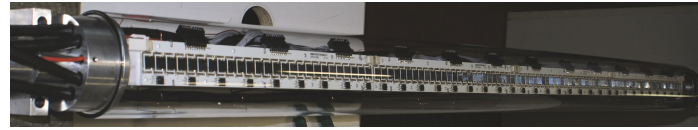

FIGURE 2: The receiver of the solar trough is made of a tube covered by a photocell row and is inside a glass tube. This is the receiver before the insertion of the CFLs.

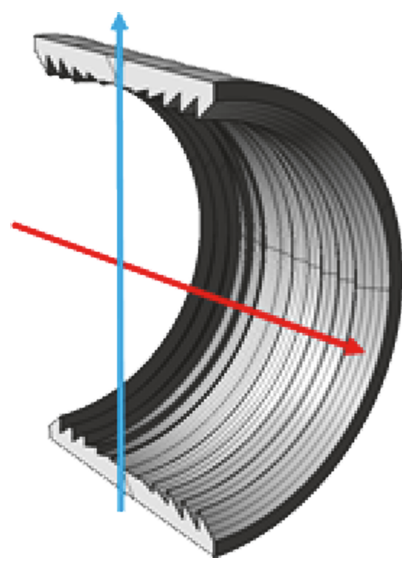

Figure 3: Perspective view of the CFL. The red arrow indicates the longitudinal axis (tube axis) and the blue arrow the transversal axis of the trough.

in a few millimeters, thus allowing to evaluate the behavior of the different sectors of the lens.

To do this, the CFL and detector are rotated around the same axis by placing them on a rotator. The detector is placed in the PV cell plane, so it is rotated together with the lens, as visualized in Figures 6 and 7. The angle of rotation is indicated by $\alpha$ in Figure 7. The axis of rotation coincides with the axis of the cylinder (red arrow in Figure 3), $25 \mathrm{~mm}$ behind the external side of the lens. The nominal focal plane of the CFL is situated at this distance.

Figures 6 and 7 illustrate the measurement scheme for two different $\alpha$ angles. By varying $\alpha$, the light strip crossing the diaphragm alternately illuminates various CFL areas.

For these tests, the sensor is a CMOS camera Vector International BCi4-6600 High Resolution USB. The camera is mounted on an $\mathrm{x}-\mathrm{y}$ translation support, separate from the lens rotation stage, which allows scanning a vertical plane. This is because the size of the image formed by the CLF is greater than the size of the camera sensor (CMOS image sensor $7.74 \times 10.51 \mathrm{~mm}$ ). In this way at each rotation of the lens, the camera had to be realigned with the lens.

An additional translator is added to vary the distance D (Figure 5). This became necessary because in visual examination, it was noted that the focal plane (i.e., the plane in which the most concentrated and uniform image was formed) was not $25 \mathrm{~mm}$ from the lens front face but slightly further. The translator is therefore necessary to perform the test at different lens-detector distances.
The procedure used to test the CFL samples is as follows:

(i) The diaphragm is adjusted to generate a $5 \mathrm{~mm}$ wide beam on the lens.

(ii) The lens is aligned in a central position $\left(\alpha=0^{\circ}\right)$.

(iii) The sensor is aligned to the lens, and three images, upper edge, center, and bottom edge of the image, are captured by moving the camera with the $\mathrm{x}-\mathrm{y}$ translator along the red arrow direction in Figure 3.

(iv) For each lens, acquisitions of the center and edge of the image were made at angles $\alpha$ between $-40^{\circ}$ and $+40^{\circ}$ at $5^{\circ}$ intervals and $\mathrm{D}$ distances between $25 \mathrm{~mm}$ and $40 \mathrm{~mm}$ at intervals of $2.5 \mathrm{~mm}$.

The laboratory set-up was also simulated with the Zemax optical simulation program, using the CFL optical design. The measurement layout is reproduced in Figure 8. The source emits a beam with solar divergence. The diaphragm is set to the same width as the one used in the laboratory tests. The detector has the same size and orientation of the CMOS camera.

As can be seen from simulations, the CFL should generate an image of about $10 \mathrm{~mm}$ in length on the PV cell (about the same size as the long side of the CMOS camera sensor). The image should have a good uniformity of lighting inside it and quite sharp edges. In fact, the laboratory tests show that

(i) the images produced by the analyzed lenses have no sharp edges and extend to a width much greater than the cell size;

(ii) the lighting has a maximum in the central part and it gradually falls towards the lateral zones;

(iii) the different lens sectors should, by symmetry, produce the same image on the camera, unless small differences are due to the inclination of the lens with respect to the diaphragm plane; instead, the images of the lateral sectors $\left(\alpha \neq 0^{\circ}\right)$ are very degraded compared to the central sector $\left(\alpha=0^{\circ}\right)$.

\section{Images Results and Discussion}

The results, as measured imaged and simulations, are illustrated for three selected samples of the last CFL production, named lens_A, lens_B, and lens_C. They are able to create (in the image plane at a suitable distance) images with better optical characteristics with respect to the previous productions. Figures 9-16 represent a selection of the most significant images measured on the three lenses, comparing each acquired image with the corresponding simulated image.

The tests performed in laboratory are done only on the secondary lens, so the concentration due to the parabolic trough is absent. When the lens is mounted on the parabolic trough, the rectangles in Figures 9-16 become squares.

Figures 9(a), 9(b), and 9(c) are images acquired at the nominal focal distance $(D=25 \mathrm{~mm})$ without rotation 


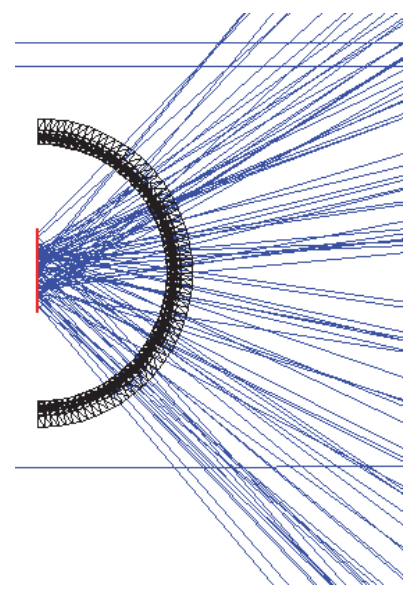

(a)

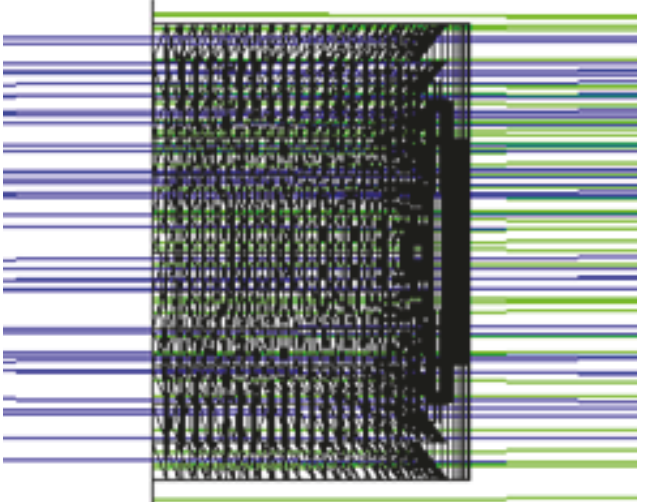

(b)

FIGURE 4: (a) Top view of the lens: the red segment represents the CFL image plane. (b) Side view of the lens: the green lines are the rays reflected by the trough.

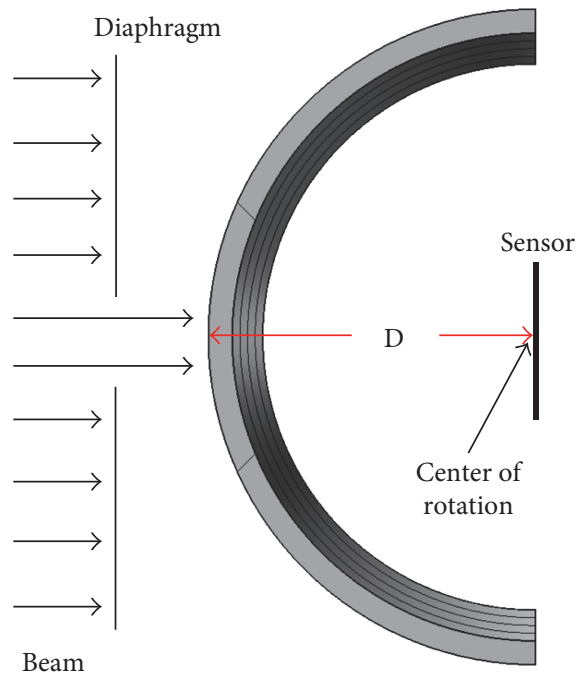

Figure 5: Top view of the lens. Lens and sensor rotate around the same axis that coincides with the CFL axis.

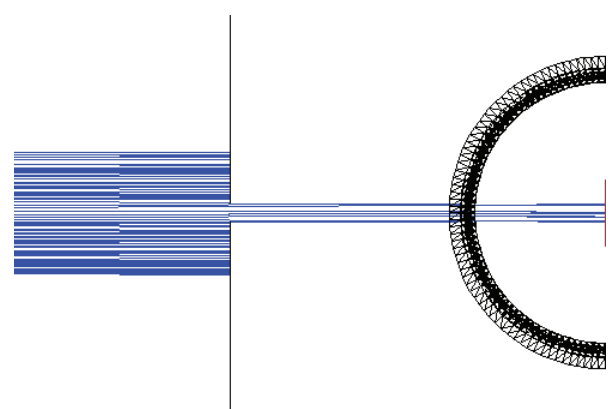

Figure 6: The light strip rays form an angle $\alpha=0^{\circ}$.

$\left(\alpha=0^{\circ}\right)$, that is, using the set-up of Figure 6. They evidence that the sample manufacturing is quite defective for lens_A, is better for lens_C, and reaches an acceptable

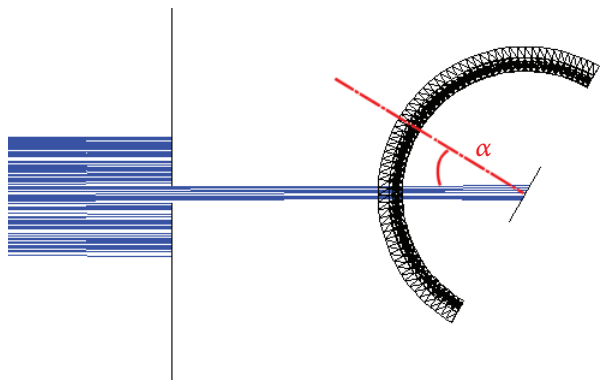

FIGURE 7: The light strip rays form an angle $\alpha=30^{\circ}$.

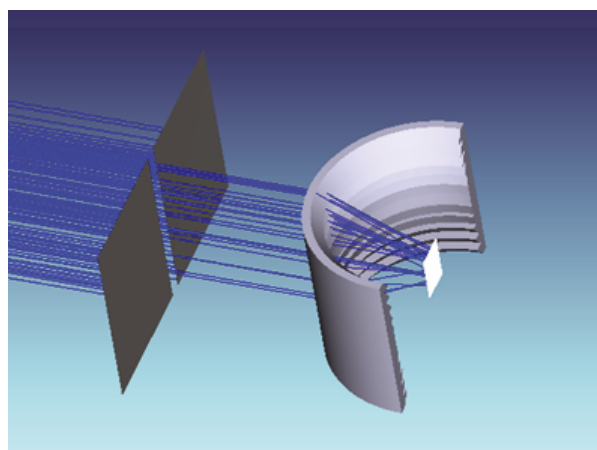

Figure 8: Zemax simulation of the layout of the laboratory set-up.

uniformity of illumination for lens_B. The related raytracing simulation gives Figure $9(\bar{d})$ as image map at $D=25 \mathrm{~mm}$ : the simulated image is in fair agreement with Figures 9(a), 9(b), and 9(c). For the simulated images, a code color is used: blue for the minimum, red for the maximum (never reached), and green is about $40 \%$ of the maximum.

Considering lens_B, Figures 10(a), 11(a), 12(a), and 13(a) were obtained from the images acquired at the 


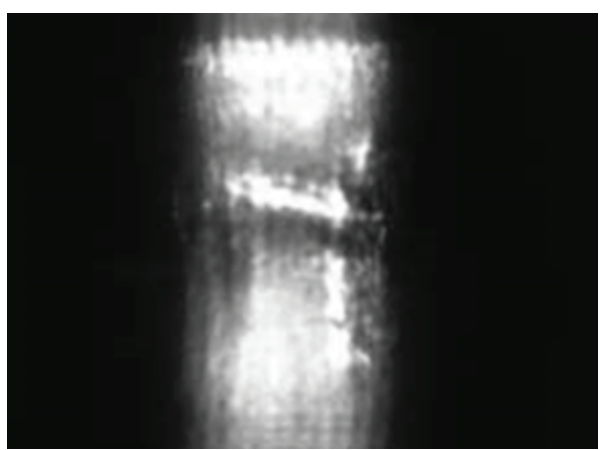

(a)

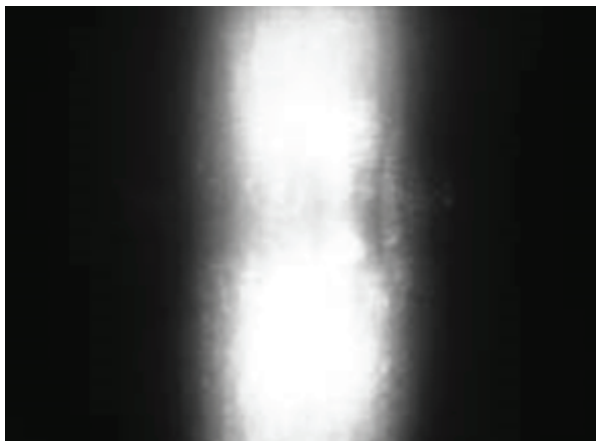

(c)

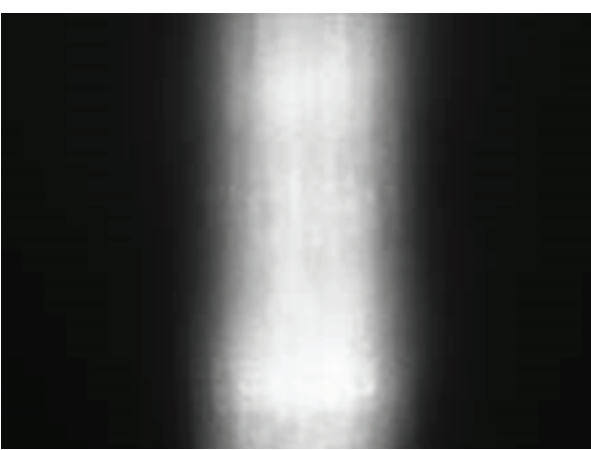

(b)

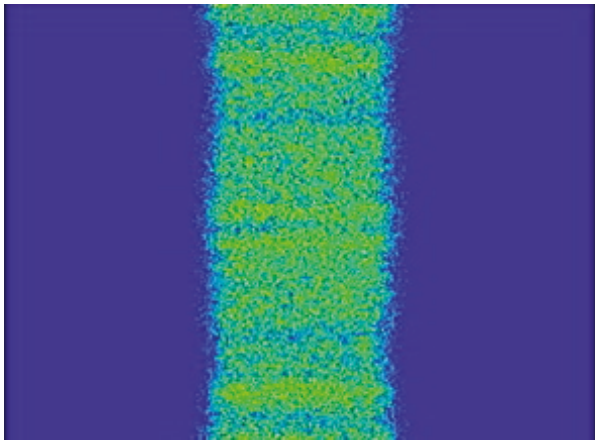

(d)

FIGURE 9: Images at $D=25 \mathrm{~mm}$ with $\alpha=0^{\circ}$ generated by lens_A (a), lens_B (b), and lens_C (c), compared with the Zemax simulation (d).

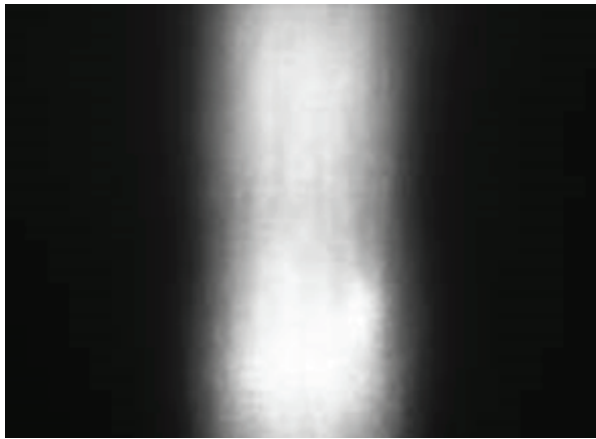

(a)

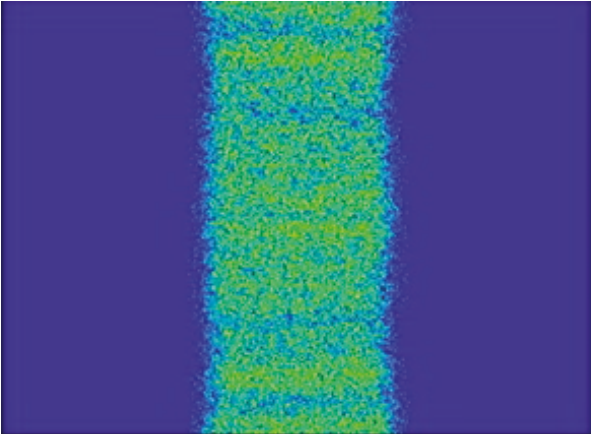

(b)

Figure 10: Images at $D=25 \mathrm{~mm}$ with $\alpha=10^{\circ}$ generated by lens_B (a), compared with the Zemax simulation (b).

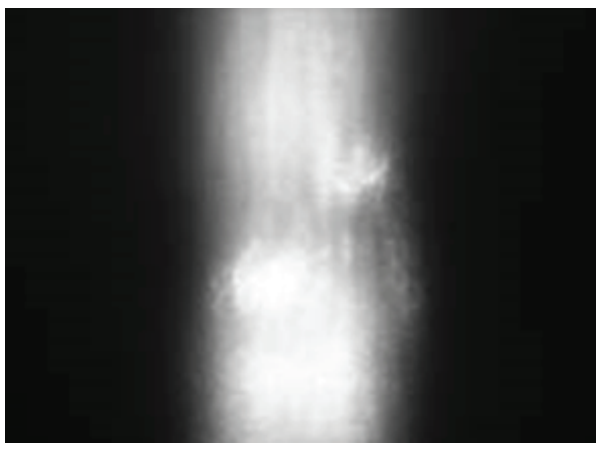

(a)

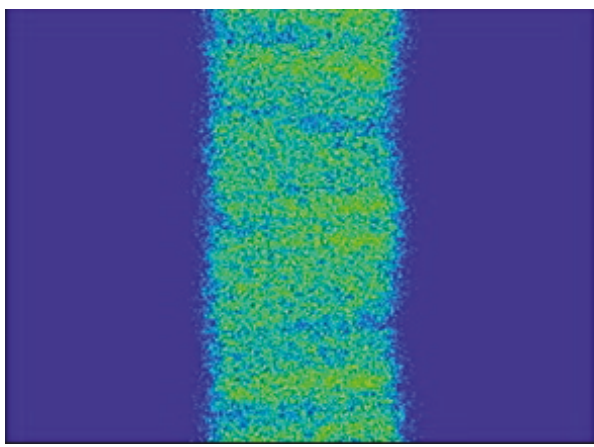

(b)

FIGURE 11: Images at $D=25 \mathrm{~mm}$ with $\alpha=20^{\circ}$ generated by lens_B (a), compared with the Zemax simulation (b). 


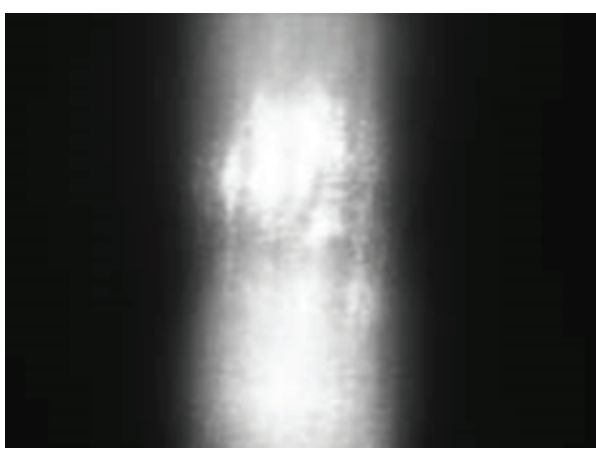

(a)

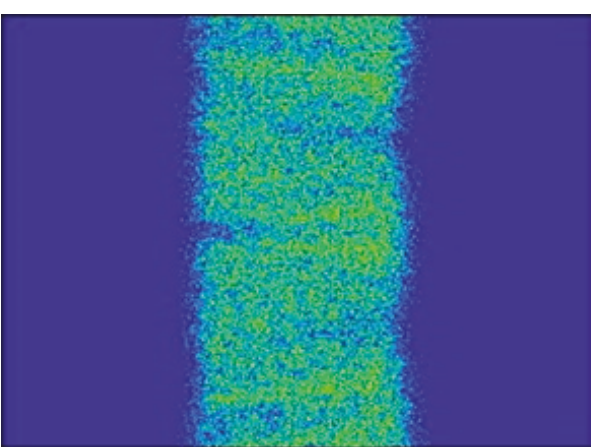

(b)

FIgURE 12: Images at $D=25 \mathrm{~mm}$ with $\alpha=30^{\circ}$ generated by lens_B (a), compared with the Zemax simulation (b).

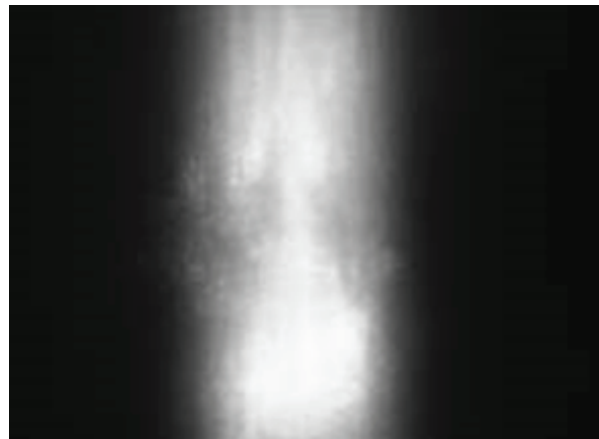

(a)

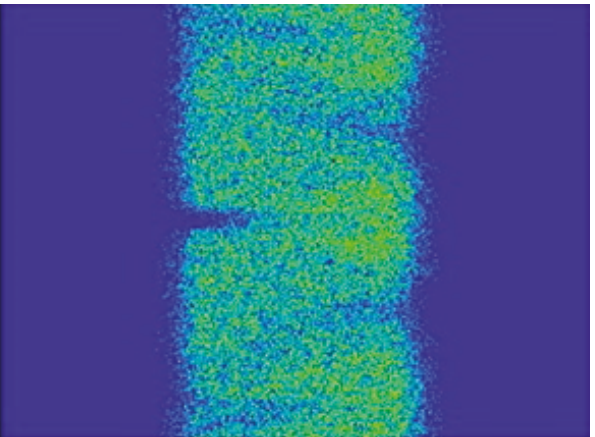

(b)

FIgURE 13: Images at $D=25 \mathrm{~mm}$ with $\alpha=40^{\circ}$ generated by lens_B (a), compared with the Zemax simulation (b).

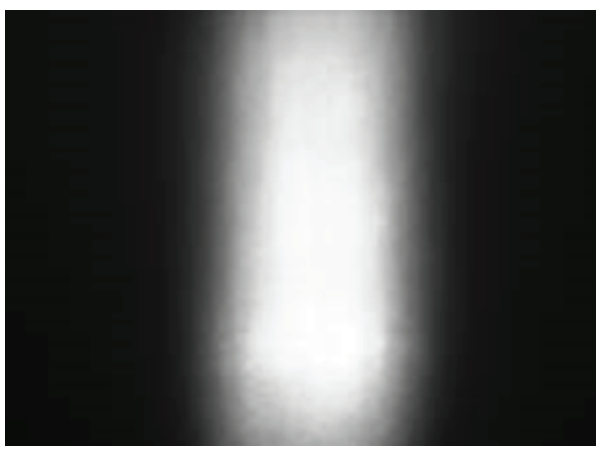

(a)

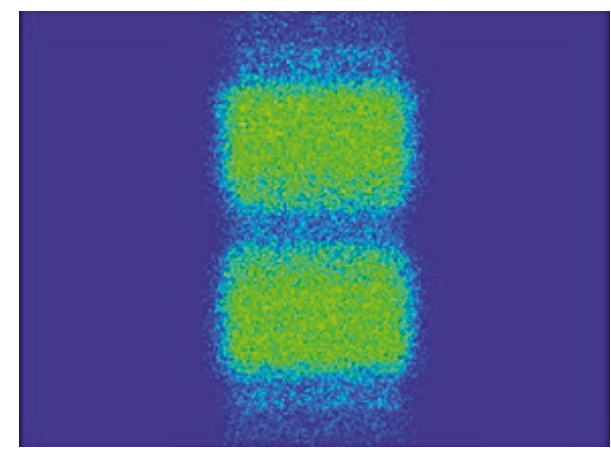

(b)

Figure 14: Images at $D=30 \mathrm{~mm}$ with $\alpha=0^{\circ}$ generated by lens_B (a), compared with the Zemax simulation (b).

nominal focal distance $(D=25 \mathrm{~mm})$ for positive $\alpha$ angles $\left(10^{\circ} \leq \alpha \leq 40^{\circ}\right)$. The images pertaining to negative $\alpha$ angles are similar and therefore are not reported. For each case examined, a Zemax simulation was performed in the corresponding configuration (the values of the parameters $\alpha$ and $D$ are indicated in the captions). The aperture width, dimension, and orientation of the detector used in the simulations correspond exactly to the set-up used in laboratory to allow direct comparison. The irradiance maps (Figures 10(b), 11(b), 12(b), and 13(b)) obtained from the simulations are shown alongside the corresponding image captured with the camera. The image distortion at angles $\alpha \neq 0$ is due to the inclination of the detector with respect to the diaphragm plane.

The principal test concerns the angular scan, so the images measured varying the $\alpha$ angle, which give information about the uniformity of manufacturing of the cylindrical lens. 


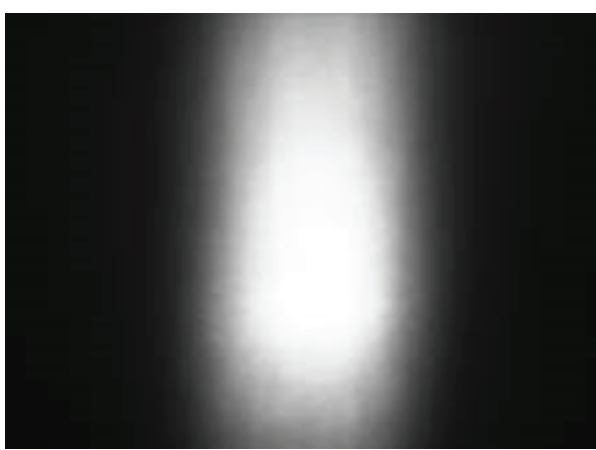

(a)

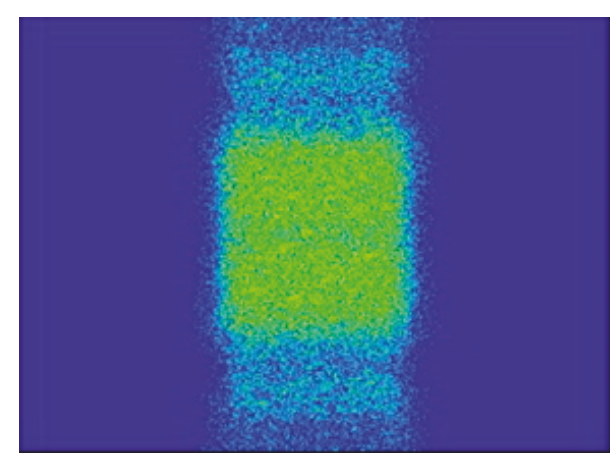

(b)

Figure 15: Images at $D=35 \mathrm{~mm}$ with $\alpha=0^{\circ}$ generated by lens_B (a), compared with the Zemax simulation (b).

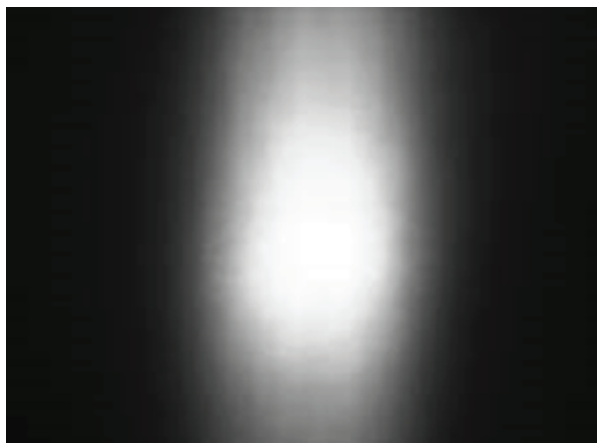

(a)

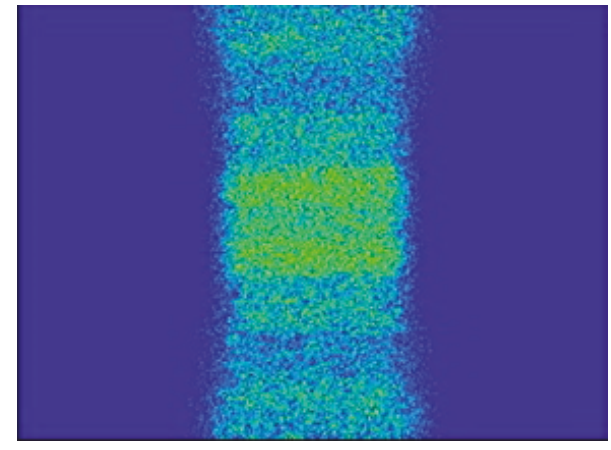

(b)

Figure 16: Images at $D=40 \mathrm{~mm}$ with $\alpha=0^{\circ}$ generated by lens_B (a), compared with the Zemax simulation (b).

On the other hand, some measurements varying the $D$ distance are useful to individuate the best focusing position to illuminate the PV cell area.

Again, choosing lens_B, Figures 14(a), 15(a), and 16(a) present acquisitions with the CFL sample in central position $\left(\alpha=0^{\circ}\right)$ and the distance $D$ variable between $30 \mathrm{~mm}$ and $40 \mathrm{~mm}$. For comparison, Figures 14(b), 15(b), and 16(b) report the irradiance maps calculated in the planes at the examined distances $D$ between lens and detector.

The main requirements concern the concentration factor and uniformity of illumination over the cell area. The concentration should be the around the value that maximizes the photovoltaic conversion of the PV cell. The collected light should be uniformly distributed over the cell area to have a good functioning of the cell. If the photocell is unevenly lighted, it does not work well and can disturb the whole cell line or even cause damage.

The three examined samples are compared in Figures 9(a), 9(b), and 9(c), and the best performing one seems to be lens_B. The results of the angular scan test on lens_B, reported in Figures 9(b), 10(a), 11(a), 12(a), and $13(\mathrm{a})\left(0^{\circ} \leq \alpha \leq 40^{\circ}\right)$, indicate that the sample contains some manufacturing imperfections, which appear as the $\alpha$ angle increases. The effect of defocusing on lens_B is analyzed in Figures 9(b), 14(a), 15(a), and 16(a): elevated levels of lighting uniformity are obtained at 25 and $30 \mathrm{~mm}$. Hence, the optimal position to place the CFL is between the nominal focal distance $(25 \mathrm{~mm})$ and $D=30 \mathrm{~mm}$.

The comparison with the irradiance maps shows a general agreement between the acquired images and the results of the Zemax ray-tracing simulations.

\section{Tailored Optical Tests: Concentration Measurements}

A quantitative assessment of the solar concentration factor was effectuated by laboratory measurements. Due to the peculiarity of the system geometry, it was chosen to calculate the concentration factor $C$ as the ratio between the density $\left(\delta_{C}\right)$ of luminous power on the cell and the power density $\left(\delta_{L}\right)$ on the lens:

$$
C=\frac{\delta_{C}}{\delta_{L}}
$$

The quantity $\delta_{C}$ is the ratio between the signal $\left(S_{C}\right)$ measured in the presence of the lens and the area $\left(a_{R}\right)$ illuminated by the receiver itself: $\delta_{C}=S_{C} / a_{R}$, while $\delta_{L}$ is the ratio between the signal $\left(S_{L}\right)$ incident on the lens and the illuminated area $\left(a_{R}\right)$ of the receiver: $\delta_{L}=S_{L} / a_{R}$. 
The illuminated area in the two cases is the same and is limited in one direction by the cell height $(10 \mathrm{~mm})$, in the other by the beam width $(3 \mathrm{~mm})$.

In conclusion,

$$
C=\frac{\delta_{C}}{\delta_{L}}=\frac{S_{C}}{S_{L}} .
$$

The set-up for the measurements is similar to the layout previously described (Figures 5-8), but with two differences.

(i) The sensor used in this case is a Hamamatsu S633701 silicon photodiode, with $18 \times 18 \mathrm{~mm}$ active area. A mask with a square aperture of $10 \mathrm{~mm}$ side was placed in front of the sensor, to limit the active area to the size of the photovoltaic cell. The center of the lens and center of the sensor are aligned with the beam direction.

(ii) The detector does not rotate solidly with the lens but is placed on a fixed support and is constantly facing in direction perpendicular to the beam, as in Figure 5. The reasons for this choice are essentially two. The first is that this procedure eliminates the uncertainty due to the fact that the sensor response is not the same at various angles of incidence. The second reason is that, as $\alpha$ changes, the determination of $\delta_{C}$ involved a factor $\cos \alpha$, because $a_{R}(\alpha)=a_{R}(\alpha=0) \times \cos \alpha$. This is not the case for $\delta_{L}$ because, due to the cylindrical symmetry, the lens illuminated area is always the same as $\alpha$ varies, and then $a_{L}(\alpha)=a_{L}(\alpha=0)$. In conclusion, the value of $C$ at different $\alpha$ would have been difficult to compare. By keeping the photodiode in a fixed position, however, it is possible to make a direct comparison of the behavior of the various lens sectors in terms of concentration factor.

The procedure used for the quantitative measurements of $C$ is as follows.

(1) The aperture width of the diaphragm is set to $3 \mathrm{~mm}$.

(2) The lens-receiver distance is set to $D=25 \mathrm{~mm}$.

(3) An angular scan is performed between $\alpha=40^{\circ}$ and $\alpha=-40^{\circ}$ at $5^{\circ}$ steps. For each angle, the signal of the photodiode $S_{C}(\alpha)$ was acquired.

(4) The lens was removed, and point (3) was repeated to capture the signal $S_{L}(\alpha)$ incident on the lens.

(5) The procedure was repeated for $D=27.5 \mathrm{~mm}$, $30 \mathrm{~mm}, 32.5 \mathrm{~mm}$, and $35 \mathrm{~mm}$.

\section{Concentration Results and Discussion}

The results of the measurement of the concentration factor $C$ are presented in Tables 1,2 , and 3 as a function of the $\alpha$ angle at the different lens-receiver distances considered. The $C$ values are discussed for three samples of the last production: lens_A, lens_B, and lens_C. Each table refers to a prototype of cylindrical Fresnel lens.

Only the concentration ratio of the secondary lens is measured, because the laboratory set-up does not reproduce the parabolic trough focalization. The value of $C$ is circa two, so when the CFL is inserted inside the solar trough, the original concentration is doubled.

The results of concentration factor $C$ are in agreement with the CLF behavior discussed in Section 4 about the images. The visual inspection shows that the best working plane is located farther than $25 \mathrm{~mm}$ (nominal focal distance). In effect, the $C$ values increase for $D$ around $30 \mathrm{~mm}$ or $32.5 \mathrm{~mm}$.

The graphs in Figures 17, 18, and 19 illustrate the trend of the $C$ concentration factor as the $\alpha$ angle changes for three samples of CFL. For more readability, only results of $D=25 \mathrm{~mm}, 30 \mathrm{~mm}$, and $35 \mathrm{~mm}$ are reported, and the other curves are among them.

The mean values of concentration factor are reported in Table 4 for each lens-receiver distance $D$ and for the three examined lenses. The maximum value of $C$ is 1.73 for lens_A, 2.13 for lens_B, and 2.09 for lens_C. The corresponding optimal positions are at $D=35 \mathrm{~mm}$ for lens_A, $D=30 \mathrm{~mm}$ for lens_B, and $D=32.5 \mathrm{~mm}$ for lens_C.

Some considerations can be done to conclude the concentration factor assessment.

(i) The theoretical concentration factor, calculated with the formulas described in Section 5 and using Zemax with the layout of Figure 8 , is $C=3.4$ for a distance $D=25 \mathrm{~mm}$. Concentration values measured in laboratory are therefore much lower than expected for the three analyzed samples. In addition, the maximum value should be recorded for $D=25 \mathrm{~mm}$ at the nominal focal length of the CFL, while in reality the maximum is found for higher distances.

(ii) The concentration factor should remain constant along the entire angular profile of the CFL. In fact, only lens_B shows such behavior.

(iii) The behavior of the three CFL samples is not the same, although the measurements have been carried out with the same procedure. Actually, the trend of $C$ for lens_A has a minimum in the center of the lens $(\alpha=0)$ and increases toward the edges; this fact is also found in the images in Section 4 , in which it can be noted that for $\alpha \approx 0$, there is a shadow area at the center of the images. For lens_C, the values are slightly lower on either side.

Test results show that the idea of adding a secondary system to perform a concentration on the longitudinal axis is both feasible and convenient. The lens construction solution, however, did not dare to get the expected performance for the CFL. The study has also provided accurate guidance on the emerging criticalities and possible solutions that will necessarily take into account the lens construction technique. 
TABLe 1: Concentration factor $C$ as a function of $\alpha$ for lens_A.

\begin{tabular}{|c|c|c|c|c|c|}
\hline$\alpha\left(^{\circ}\right)$ & $D=25 \mathrm{~mm}$ & $D=27.5 \mathrm{~mm}$ & $D=30 \mathrm{~mm}$ & $D=32.5 \mathrm{~mm}$ & $D=35 \mathrm{~mm}$ \\
\hline 40 & 1.56 & 1.67 & 1.77 & 1.84 & 1.87 \\
\hline 35 & 1.51 & 1.64 & 1.74 & 1.83 & 1.86 \\
\hline 30 & 1.46 & 1.58 & 1.69 & 1.80 & 1.84 \\
\hline 25 & 1.45 & 1.53 & 1.62 & 1.74 & 1.79 \\
\hline 20 & 1.42 & 1.51 & 1.59 & 1.68 & 1.71 \\
\hline 15 & 1.38 & 1.46 & 1.54 & 1.62 & 1.65 \\
\hline 10 & 1.36 & 1.45 & 1.51 & 1.56 & 1.58 \\
\hline 5 & 1.35 & 1.44 & 1.49 & 1.54 & 1.57 \\
\hline 0 & 1.35 & 1.44 & 1.49 & 1.54 & 1.56 \\
\hline-5 & 1.34 & 1.42 & 1.48 & 1.54 & 1.56 \\
\hline-10 & 1.36 & 1.45 & 1.51 & 1.57 & 1.60 \\
\hline-15 & 1.39 & 1.47 & 1.55 & 1.61 & 1.65 \\
\hline-20 & 1.42 & 1.52 & 1.59 & 1.67 & 1.72 \\
\hline-25 & 1.46 & 1.56 & 1.65 & 1.74 & 1.80 \\
\hline-30 & 1.52 & 1.63 & 1.73 & 1.83 & 1.87 \\
\hline-35 & 1.58 & 1.69 & 1.81 & 1.89 & 1.89 \\
\hline-40 & 1.65 & 1.76 & 1.84 & 1.90 & 1.90 \\
\hline
\end{tabular}

TABLE 2: Concentration factor $C$ as a function of $\alpha$ for lens_B.

\begin{tabular}{|c|c|c|c|c|c|}
\hline$\alpha\left(^{\circ}\right)$ & $D=25 \mathrm{~mm}$ & $D=27.5 \mathrm{~mm}$ & $D=30 \mathrm{~mm}$ & $D=32.5 \mathrm{~mm}$ & $D=35 \mathrm{~mm}$ \\
\hline 40 & 1.96 & 2.03 & 2.07 & 2.07 & 2.05 \\
\hline 35 & 1.98 & 2.05 & 2.09 & 2.10 & 2.06 \\
\hline 30 & 1.98 & 2.06 & 2.11 & 2.12 & 2.08 \\
\hline 25 & 2.00 & 2.07 & 2.11 & 2.11 & 2.09 \\
\hline 20 & 2.02 & 2.11 & 2.15 & 2.13 & 2.10 \\
\hline 15 & 2.00 & 2.11 & 2.16 & 2.16 & 2.13 \\
\hline 10 & 1.98 & 2.07 & 2.13 & 2.13 & 2.10 \\
\hline 5 & 1.97 & 2.06 & 2.10 & 2.10 & 2.08 \\
\hline 0 & 2.00 & 2.09 & 2.15 & 2.15 & 2.13 \\
\hline-5 & 2.03 & 2.13 & 2.19 & 2.19 & 2.16 \\
\hline-10 & 2.04 & 2.13 & 2.18 & 2.17 & 2.13 \\
\hline-15 & 2.02 & 2.11 & 2.17 & 2.17 & 2.15 \\
\hline-20 & 2.03 & 2.13 & 2.18 & 2.17 & 2.14 \\
\hline-25 & 2.02 & 2.11 & 2.15 & 2.15 & 2.12 \\
\hline-30 & 2.01 & 2.08 & 2.13 & 2.13 & 2.10 \\
\hline-35 & 1.99 & 2.07 & 2.12 & 2.12 & 2.07 \\
\hline-40 & 2.00 & 2.07 & 2.10 & 2.09 & 2.05 \\
\hline
\end{tabular}

\section{Conclusion}

The purpose was to develop a secondary optics for a concentrating photovoltaic trough that increases the solar concentration and reduces the photovoltaic cell number. The proposed cylindrical Fresnel lens (CFL) focuses in the direction where the trough does not concentrate: in practice, it transforms the focal line into a series of focal points.

Some lens prototypes were produced based on the CFL optical design. Tailored tests were developed to optically characterize and check the quality of the manufactured CFL samples [12]. The performed tests help to localize the zones with defects, so to improve the lens production.

The image analysis gives a qualitative assessment of the CFL quality: every image measured on a lens indicates the level of uniformity of sun concentration, which is fundamental to correctly exploit the solar cells. The behavior of each examined lens was simulated using a ray-tracing software (Zemax), and the simulated CFL image is in general agreement with the measured image, but some exceptions are presented.

Quantitative measurements assessed the concentration factor $C$, for various angles along the curve lens and for 
TABLe 3: Concentration factor $C$ as a function of $\alpha$ for lens_C.

\begin{tabular}{|c|c|c|c|c|c|}
\hline$\alpha\left(^{\circ}\right)$ & $D=25 \mathrm{~mm}$ & $D=27.5 \mathrm{~mm}$ & $D=30 \mathrm{~mm}$ & $D=32.5 \mathrm{~mm}$ & $D=35 \mathrm{~mm}$ \\
\hline 40 & 1.88 & 1.98 & 2.02 & 2.03 & 1.97 \\
\hline 35 & 1.90 & 1.98 & 2.03 & 2.05 & 2.01 \\
\hline 30 & 1.91 & 1.99 & 2.04 & 2.05 & 2.02 \\
\hline 25 & 1.92 & 2.00 & 2.04 & 2.06 & 2.01 \\
\hline 20 & 1.91 & 2.00 & 2.04 & 2.05 & 2.00 \\
\hline 15 & 1.88 & 1.98 & 2.02 & 2.05 & 2.01 \\
\hline 10 & 1.87 & 1.97 & 2.01 & 2.01 & 1.98 \\
\hline 5 & 1.87 & 1.95 & 1.99 & 2.00 & 1.96 \\
\hline 0 & 1.92 & 2.02 & 2.06 & 2.08 & 2.03 \\
\hline-5 & 1.95 & 2.06 & 2.10 & 2.11 & 2.07 \\
\hline-10 & 1.97 & 2.07 & 2.12 & 2.14 & 2.09 \\
\hline-15 & 2.01 & 2.11 & 2.15 & 2.14 & 2.10 \\
\hline-20 & 2.03 & 2.13 & 2.16 & 2.14 & 2.09 \\
\hline-25 & 2.05 & 2.13 & 2.16 & 2.15 & 2.09 \\
\hline-30 & 2.05 & 2.12 & 2.14 & 2.14 & 2.11 \\
\hline-35 & 2.03 & 2.10 & 2.15 & 2.18 & 2.08 \\
\hline-40 & 2.05 & 2.14 & 2.16 & 2.15 & 2.10 \\
\hline
\end{tabular}

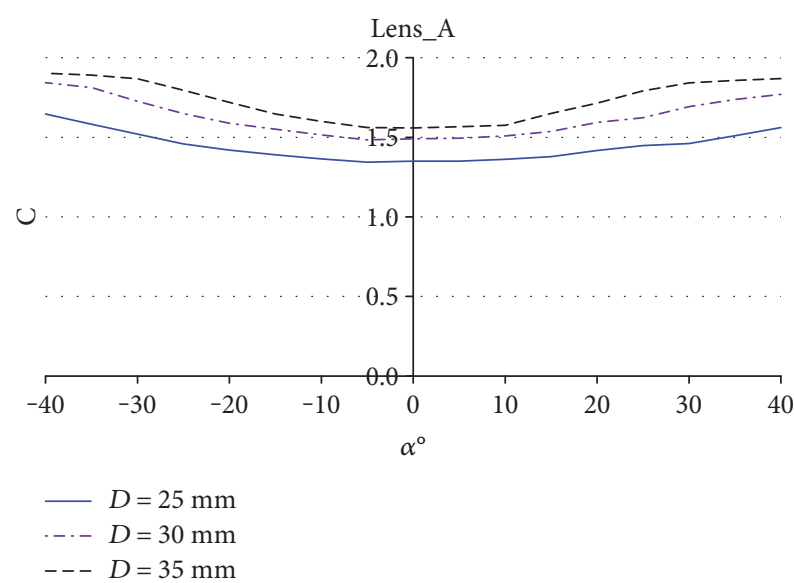

FIgURE 17: Concentration factor $C$ as a function of $\alpha$ for lens_A.

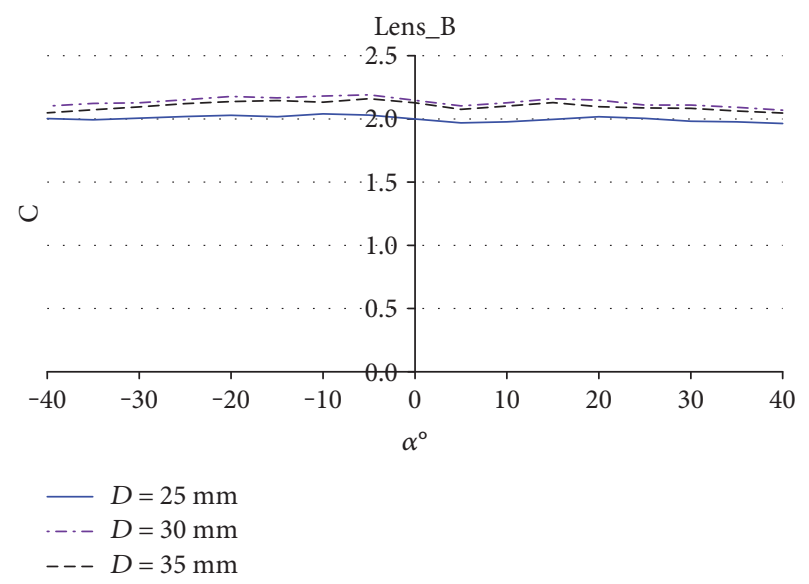

FIgURE 18: Concentration factor $C$ as a function of $\alpha$ for lens_B.

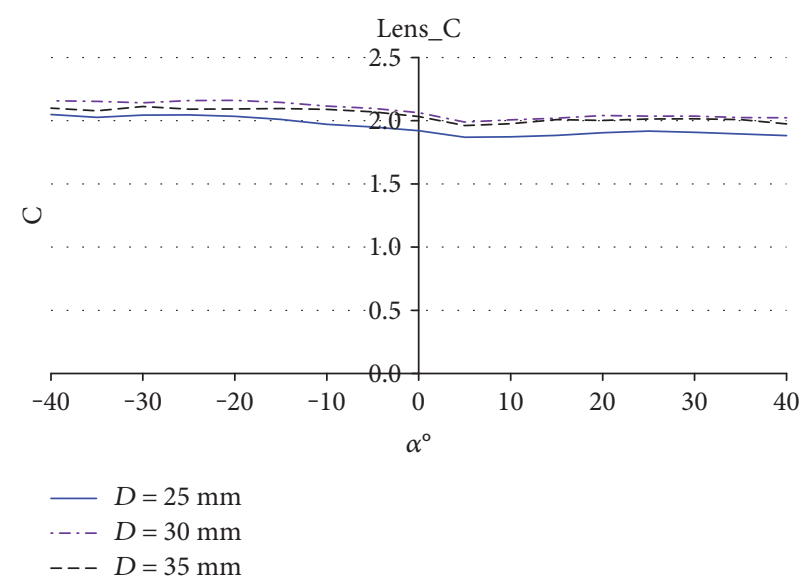

Figure 19: Concentration factor $C$ as a function of $\alpha$ for lens_C.

different lens-cell distances $D$. The maxima of average $C$ are 1.73 for lens_A, 2.13 for lens_B, and 2.09 for lens_C, respectively, obtained at D $35 \mathrm{~mm}, 30 \mathrm{~mm}$, and $32.5 \mathrm{~mm}$. For each lens, $C$ varies depending on $D$, so the $C$ maximum corresponds to the best distance $D$ for placing the cell.

The trough concentration ratio before the introduction of the secondary optics was 140; since the CFL doubles it, $C$ approaches 300 for the system with primary mirror and secondary lens. This $C$ value allows to utilize the original PV cells and avoids the need to employ more expensive multijunction cells.

The optical performance improves with the successive CFL productions. The three selected samples reach a higher concentration factor and create images with better optical characteristics with respect to the previous products. In spite of this, they do not meet the requirements in terms of concentration and lighting uniformity on the cell plane (in fact 
TABLE 4: Mean values of the concentration factor $C$.

\begin{tabular}{|c|c|c|c|c|c|}
\hline Lens & $D=25 \mathrm{~mm}$ & $D=27.5 \mathrm{~mm}$ & $D=30 \mathrm{~mm}$ & $D=32.5 \mathrm{~mm}$ & $D=35 \mathrm{~mm}$ \\
\hline Lens_A & 1.44 & 1.54 & 1.62 & 1.70 & 1.73 \\
\hline Lens_B & 2.00 & 2.09 & 2.13 & 2.12 & 2.10 \\
\hline Lens_C & 1.95 & 2.04 & 2.08 & 2.09 & 2.04 \\
\hline
\end{tabular}

the $C$ maximum is at different $D$ ), so the manufacturing process needs further improvements.

The study and the tests have made possible to detect the criticality of the first CFL prototypes, and they provide some precise indications of the possible actions to be implemented, also considering a rethinking of the lens construction processes adopted, which can be considered as the main responsible for the loss of optical lens performance.

\section{Conflicts of Interest}

The authors declare that they have no conflicts of interest.

\section{References}

[1] D. Fontani, P. Sansoni, F. Francini, and D. Jafrancesco, Designing an Innovative Secondary Optics for Parabolic Trough, Proceedings of SWC2015, Daegu, Korea, International Solar Energy Society, 2016.

[2] D. W. Kearney, Parabolic Trough Collector Overview, Parabolic Trough Workshop 2007 at the National Renewable Energy Laboratory, Golden CO, 2007, http://www.doc88. $\mathrm{com} / \mathrm{p}-882640289219 . \mathrm{html}$.

[3] D. Krüger, Y. Pandian, K. Hennecke, and M. Schmitz, "Parabolic trough collector testing in the frame of the REACt project," Desalination, vol. 220, no. 1-3, pp. 612-618, 2008.

[4] G. C. Bakos, I. Ioannidis, N. F. Tsagas, and I. Seftelis, "Design, optimisation and conversion-efficiency determination of a line-focus parabolic-trough solar-collector (PTC)," Applied Energy, vol. 68, no. 1, pp. 43-50, 2000.

[5] H. Price, E. Lüpfert, D. Kearney et al., "Advances in parabolic trough solar power technology," Journal of Solar Energy Engineering, vol. 124, no. 2, pp. 109-125, 2002.

[6] D. E. Prapas, B. Norton, and S. D. Probert, "Optics of parabolic-trough, solar-energy collectors, possessing small concentration ratios," Solar Energy, vol. 39, no. 6, pp. 541-550, 1987.

[7] W. Weiss and M. Rommel, Solar Heat for Industrial Process: State of the Art-Medium Temperature Collectors, IEA-SHC Task 33/IV, 2005.

[8] J. Klapp, J. L. Cervantes-Cota, and J. F. Alcalá Chávez, Towards a Cleaner Planet: Energy for the Future, Springer, 2007.

[9] T. C. Kandpal, S. S. Mathur, and A. K. Singhal, "Optical performance of a composite parabolic trough," Applied Energy, vol. 19, no. 3, pp. 231-239, 1985.

[10] H. M. Güven and R. B. Bannerot, "Determination of error tolerances for the optical design of parabolic troughs for developing countries," Solar Energy, vol. 36, no. 6, pp. 535-550, 1986.

[11] R. Winston, J. C. Miñano, P. Benítez, N. Shatz, and J. C. Bortz, Nonimaging Optics, Elsevier Academic Press, Amsterdam, 2005.
[12] D. Fontani, F. Francini, and P. Sansoni, "Optical characterisation of solar concentrator," Optics and Lasers in Engineering, vol. 45, pp. 351-359, 2007.

[13] D. Fontani, P. Sansoni, E. Sani, S. Coraggia, D. Jafrancesco, and L. Mercatelli, "Solar divergence collimators for optical characterisation of solar components," International Journal of Photoenergy, vol. 2013, Article ID 610173, 10 pages, 2013. 

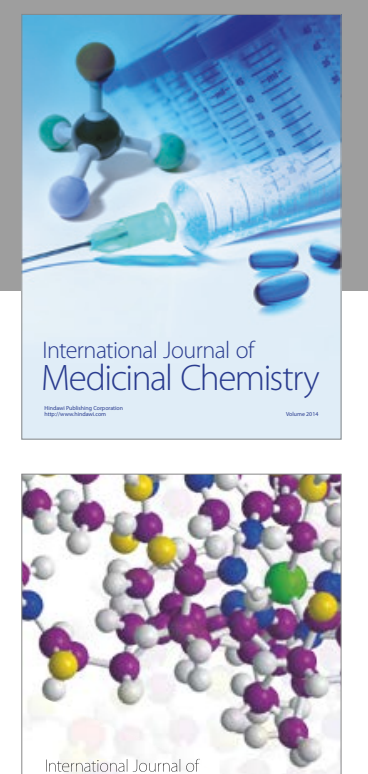

Carbohydrate Chemistry

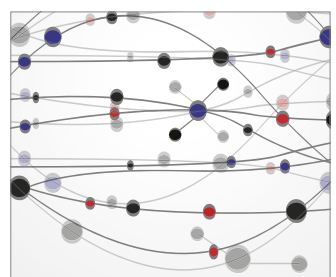

The Scientific World Journal
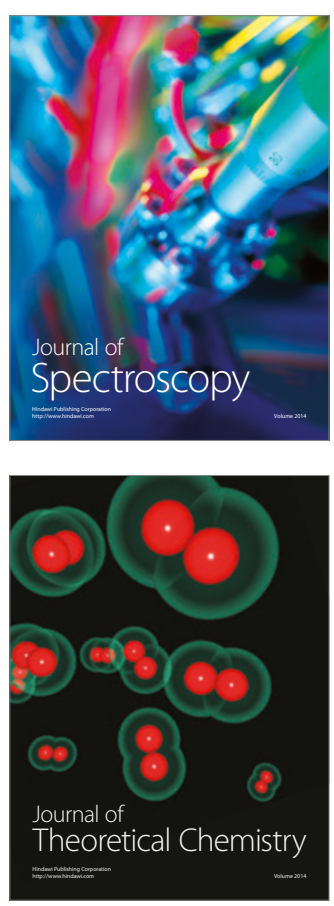
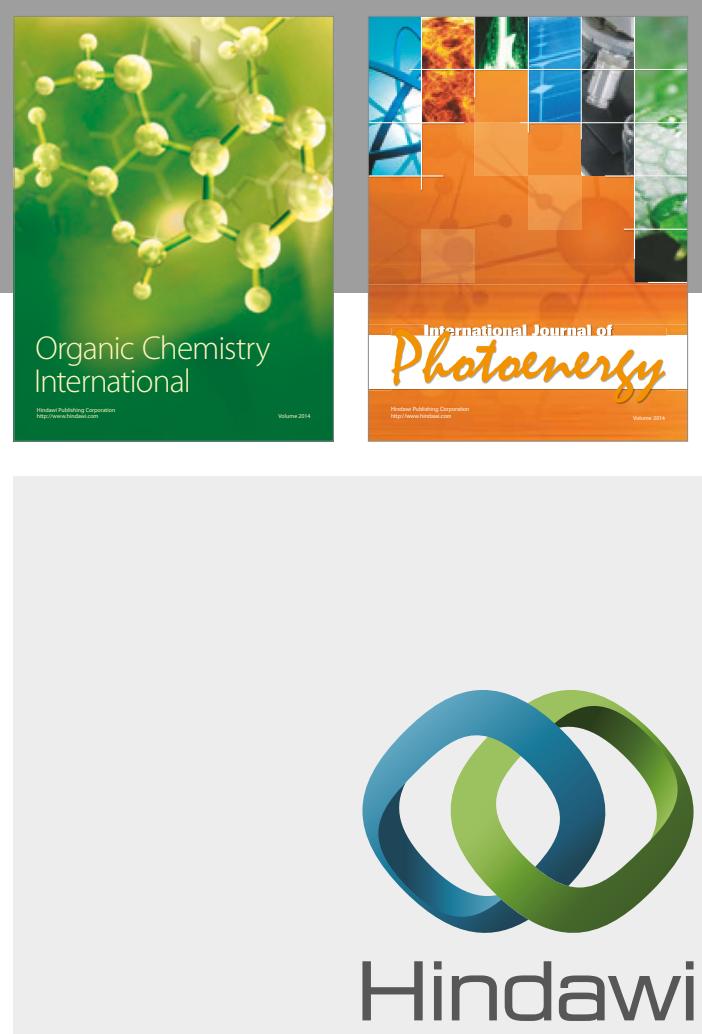

Submit your manuscripts at

https://www.hindawi.com

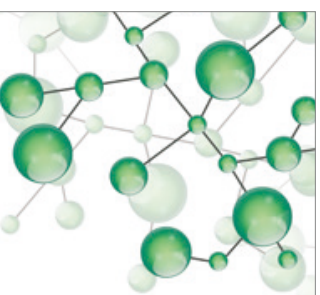

International Journal of

Inorganic Chemistry

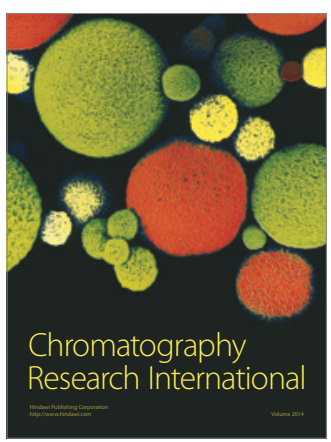

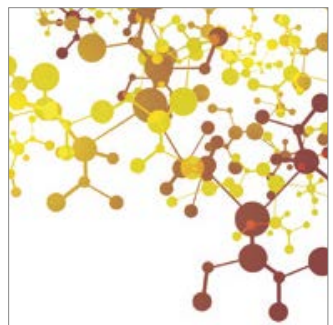

Applied Chemistry
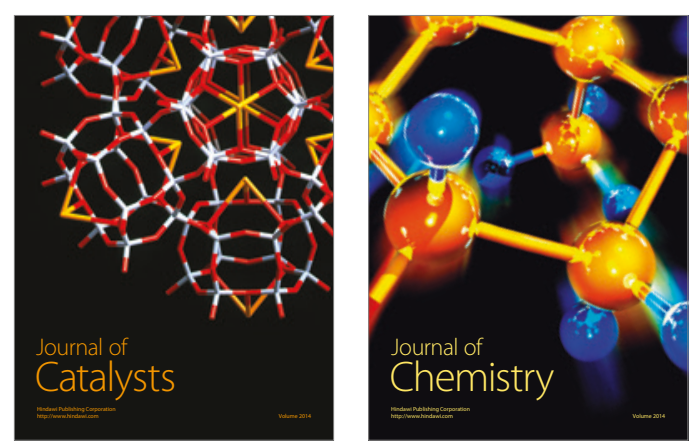
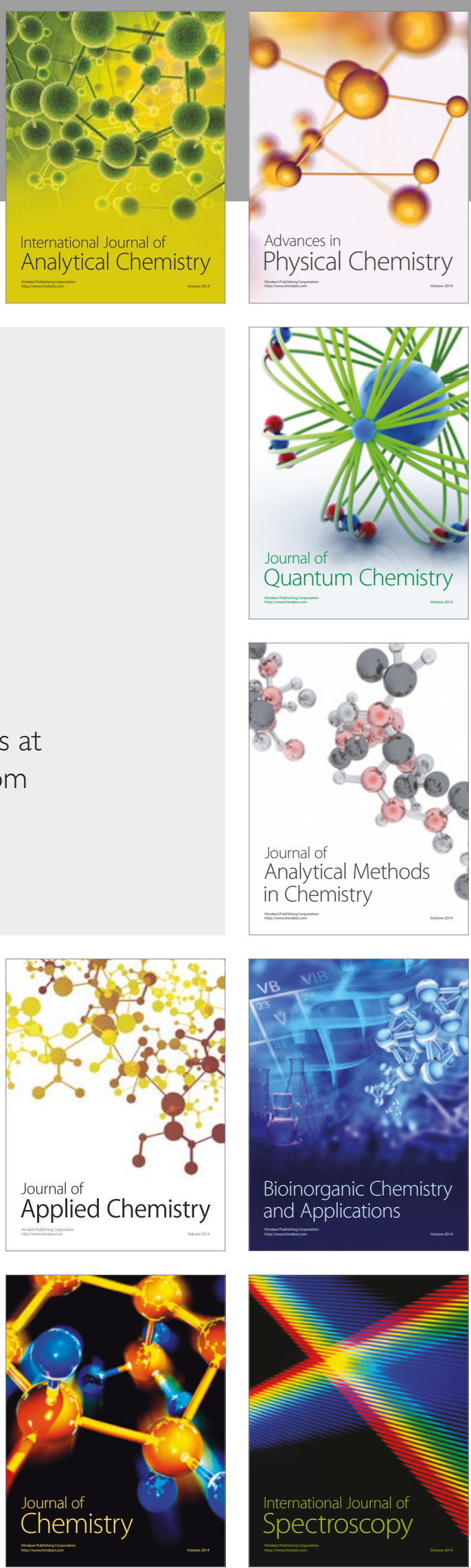